\title{
Regular homotopy and total curvature II: sphere immersions into 3-space
}

\author{
TOBIAS EKHOLM
}

\begin{abstract}
We consider properties of the total curvature functional on the space of 2-sphere immersions into 3-space. We show that the infimum over all sphere eversions of the maximum of the total curvature during an eversion is at most $8 \pi$ and we establish a non-injectivity result for local minima.
\end{abstract}

$53 \mathrm{C} 42 ; 53 \mathrm{~A} 04,57 \mathrm{R} 42$

\section{Introduction}

An immersion of manifolds is a map with everywhere injective differential. Two immersions are regularly homotopic if there exists a continuous 1-parameter family of immersions connecting one to the other. The Smale-Hirsch $h$-principle [10; 4] says that the space of immersions $M \rightarrow N$, $\operatorname{dim}(M)<\operatorname{dim}(N)$ is homotopy equivalent to the space of injective bundle maps $T M \rightarrow T N$. In contrast to differential topological properties, differential geometric properties of immersions do not in general satisfy $h$-principles, see [3, (A) on page 62]. In this paper and the predecessor [2], we study some aspects of the differential geometry of immersions and regular homotopies in the most basic cases of codimension one immersions. We investigate whether or not it is possible to perform topological constructions while keeping control of certain geometric quantities.

Consider immersions $S^{2} \rightarrow \mathbb{R}^{3}$. In this case the $h$-principle implies a famous theorem of Smale [9]: all immersions $S^{2} \rightarrow \mathbb{R}^{3}$ are regularly homotopic. In particular, there exists sphere eversions (ie, regular homotopies connecting the unit 2 -sphere in $\mathbb{R}^{3}$ to the same immersion with the opposite (co)orientation).

The total curvature $\kappa(f)$ of a sphere immersion $f: S^{2} \rightarrow \mathbb{R}^{3}$ is the mapping area of its Gauss map:

$$
\kappa(f)=\int_{S^{2}}|K| d \sigma,
$$

where $K$ is the Gaussian curvature and $d \sigma$ the area element induced on $S^{2}$ by the immersion $f$. The functional $\kappa$ (and its higher dimensional generalizations) is also 
known as the Lipschitz-Killing curvature. It follows from the Gauss-Bonnet theorem that the global minimum of $\kappa$ equals $4 \pi$. An immersion for which this value is attained is called tight (or convex) and is known to be the boundary of a convex body in $\mathbb{R}^{3}$, Kuiper [5]. This characterization of tight immersions immediately implies that any sphere eversion $f_{t}, 0 \leq t \leq 1$, has an instant $f_{\tau}$ with $\kappa\left(f_{\tau}\right)>4 \pi$. The following result gives a corresponding upper bound.

Theorem 1.1 For every $\epsilon>0$ there exists a sphere eversion $f_{t}, 0 \leq t \leq 1$, with

$$
\max _{0 \leq t \leq 1} \kappa\left(f_{t}\right)<8 \pi+\epsilon .
$$

In fact, $f_{t}$ can be chosen so that for each $t \in[0,1]$ there exists a unit vector $v_{t} \in \mathbb{R}^{3}$ such that the height function $\left\langle f_{t}, v_{t}\right\rangle: S^{2} \rightarrow \mathbb{R}$ has exactly two non-degenerate critical points.

Theorem 1.1 is proved in Section 2.2. It is interesting to compare this result to the fact that the $L^{2}$-norm of the mean curvature $W(f)$ (also known as the Willmore energy) of immersions $f: S^{2} \rightarrow \mathbb{R}^{3}$ has the following property. For any sphere eversion $f_{t}$, $0 \leq t \leq 1$, there exists some $\tau \in(0,1)$ such that $W\left(f_{\tau}\right) \geq 16 \pi$. (This is a consequence of two results: any sphere eversion has a quadruple point, Max-Banchoff [8], and $W(f) \geq 16 \pi$ for any immersion $f$ with a quadruple point, Li-Yau [7].)

It is unknown to the author whether the result in Theorem 1.1 is best possible. We therefore ask: what is the infimum of $\max _{0 \leq t \leq 1} \kappa\left(f_{t}\right)$ over all sphere eversions $f_{t}$, $0 \leq t \leq 1$ ?

In Propositions 3.3 and 3.4 we make two general observations about local minima of $\kappa$ : an immersion of a closed $n$-manifold into $\mathbb{R}^{n+1}$ which is a critical point of $\kappa$ must have total curvature a multiple of the volume of the unit $n$-sphere and its Gauss map cannot have fold singularities. These observations imply that all local minima of $\kappa$ with curvature function which meets a non-degeneracy condition are so called relatively isotopy tight (RIT) immersions with certain special properties, see Section 4.2. (Relatively isotopy tight immersions were introduced by Kuiper and Meeks in [6], we recall their definition in Section 4.1.) We show in Theorem 4.2 that RIT immersions with the special properties just mentioned are non-injective. As a consequence we obtain the following result.

Theorem 1.2 Any local minimum $f: S^{2} \rightarrow \mathbb{R}^{3}$ of $\kappa$ with 0 a non-trivial regular value of $K$ is an RIT immersion such that any component of $K^{-1}((-\infty, 0])$ is an annulus. In particular, no such local minimum is an embedding.

Theorem 1.2 is proved in Section 4.3.

Algebraic 83 Geometric Topology, Volume 6 (2006) 


\section{Acknowledgements}

During the preparation of this paper, the author was a research fellow of the Royal Swedish Academy of Sciences sponsored by the Knut and Alice Wallenberg foundation. He also acknowledges support from the Alfred P Sloan Foundation and from NSF-grant DMS-0505076.

\section{Total curvature and a sphere eversion}

In this section we recall properties of the total curvature of a closed $n$-manifold immersed into $\mathbb{R}^{n+1}$. We then describe a sphere eversion which constitutes a proof of Theorem 1.1.

\subsection{Total curvature}

Let $f: M \rightarrow \mathbb{R}^{n+1}$ be an immersion of a closed oriented $n$-dimensional manifold. Let $v: M \rightarrow \mathbb{S}^{n}$, where $\mathbb{S}^{n}$ is the unit sphere in $\mathbb{R}^{n+1}$, be its Gauss map which takes a point $p \in M$ to the positive unit normal of $d f\left(T_{p} M\right)$. The total curvature $\kappa(f)$ of $f$ is the mapping area of $v$. Standard results, see Cecil and Ryan [1], then imply

$$
\kappa(f)=\int_{M}|J(v)| d \sigma=\frac{1}{2} \int_{\mathbb{S}^{n}} \mu\left(f_{v}\right) d v,
$$

where in the first expression $|J(v)|$ is the absolute value of the Gauss-Kronecker curvature of $f$ and $d \sigma$ is the volume element of the metric induced by $f$, and where in the second, integral geometric expression, $\mu\left(f_{v}\right)$ denotes the number of critical points of the function $f_{v}: M \rightarrow \mathbb{R}, f_{v}(x)=\langle f(x), v\rangle$, where $v \in \mathbb{S}^{n}$ is a unit vector.

\subsection{A sphere eversion}

Let $f: S^{2} \rightarrow \mathbb{R}^{3}$ be any immersion. Then for generic unit vectors $v$ in $\mathbb{R}^{3}$ the composition of $f$ with the orthogonal projection $\pi_{v}$ along $v$ to a plane perpendicular to $v$ is a map with only stable singularities. In particular this means that the image of the singular set of $\pi_{v} \circ f$ is a piecewise regular curve with cusp singularities. More precisely, the jet transversality theorem implies that for generic $v \in \mathbb{S}^{2}$ the set of points $p \in S^{2}$ such that $v \in d f\left(T_{p} S^{2}\right)$ is a smooth 1-dimensional submanifold $\Sigma_{v}(f) \subset S^{2}$ and that $v$ gives a vector field along $\Sigma_{v}(f)$ which is tangent to $\Sigma_{v}(f)$ with order one tangencies at isolated points in $\Sigma_{v}(f)$. These points form a subset which we denote $\Sigma_{v}^{\prime}(f) \subset \Sigma_{v}(f)$. We call the image of the singular set $\Sigma_{v}(f)$ under $\pi_{v} \circ f$ the fold curve of $\pi_{v} \circ f$. It is a piecewise regular curve with singularities 
corresponding to the points in $\Sigma_{v}^{\prime}(f)$, where the fold curve looks like a semi-cubical cusp $\left\{(x, y) \in \mathbb{R}^{2}: x^{2}=y^{3}\right\}$ up to left-right action of diffeomorphisms of the plane, we call such singularities cusps. Define the total curvature $\kappa$ of a piecewise regular planar curve with cusps as the sum of the total curvatures $\int_{c}|k| d s$ of its regular pieces $c$, where $k$ is the curvature function of the regular curve $c$. (Note that there are no curvature concentrations at the cusps.) We then have the following

Lemma 2.1 If $f: S^{2} \rightarrow \mathbb{R}^{3}$ is an immersion then

$$
\kappa(f)=\frac{1}{2 \pi} \int_{v \in \mathbb{S}^{2}} \kappa\left(\pi_{v} \circ f\left(\Sigma_{v}(f)\right)\right) d v .
$$

Proof This is immediate from the integral geometric expression for the total curvature: the local extrema of the height function $f_{w}$ for $w$ perpendicular to $v$ are in one to one correspondence with the local extrema of the height function in direction $w$ on the curve $\pi \circ f\left(\Sigma_{v}(f)\right)$ which are not cusps, see (2-2) below. This implies that (2-1) holds up to an over all normalizing constant. Considering the round sphere it is easy to see that this constant equals $\frac{1}{2 \pi}$ as claimed.

Consider an immersion $f: S^{2} \rightarrow \mathbb{R}^{3}$ and let $v$ be a generic unit vector. Thinking of $\mathbb{R}^{3}$ as $\mathbb{R}^{2} \times \mathbb{R}$, where $v$ points in the $\mathbb{R}$-direction we write $f=\left(\pi_{v} \circ f, f_{v}\right)$. For $\lambda>0$ the map $f^{\lambda, v}=\left(\pi_{v} \circ f, \lambda f_{v}\right)$ is an immersion.

Lemma 2.2 As $\lambda \rightarrow 0, \kappa\left(f^{\lambda, v}\right)$ tends to twice the total curvature of the fold curve of $\pi_{v} \circ f$. As $\lambda \rightarrow \infty, \kappa\left(f^{\lambda, v}\right)$ tends to $2 \pi$ times the number of critical points of the Morse function $f_{v}$.

Proof The first statement is a consequence of Lemma 2.1 together with the fact if $w \neq \pm v$ is any unit vector, $w=\alpha v+\beta w_{0},\left\langle w_{0}, v\right\rangle=0,\left\langle w_{0}, w_{0}\right\rangle=1$, and $\beta>0$, then $f^{\lambda, v}{ }_{w} \rightarrow f_{w_{0}}$ as $\lambda \rightarrow 0$. The second statement follows similarly from the integral geometric formula for the total curvature.

We will next construct a sphere eversion $f_{t}: S^{2} \rightarrow \mathbb{R}^{3}, 0 \leq t \leq 1$, such that $\max _{0 \leq t \leq 1} \kappa\left(f_{t}\right) \leq 8 \pi+\epsilon$. To this end we first describe the middle stage $g_{\frac{1}{2}}$ of a sphere eversion $g_{t}: S^{2} \rightarrow \mathbb{R}^{3}, 0 \leq t \leq 1$, closely related to $f_{t}$. This middle stage is a nearly planar immersion: $g_{\frac{1}{2}}=\left(\pi_{v} \circ h, \lambda h_{v}\right)$ for some immersion $h$ and for small $\lambda>0$. In Figure 1 we depict the image of the projection $\pi_{v} \circ h$. In order to see that Figure 1 is really the projection of a sphere immersion we argue as follows. Subdivide $S^{2}$ into three parts: an thin band $B$ around the equator, a northern disk $D_{n}$, and a southern disk $D_{s}$. Figure 2 depicts the images under $\pi_{v} \circ h$ of $D_{n}$ and $D_{s}$. In order 


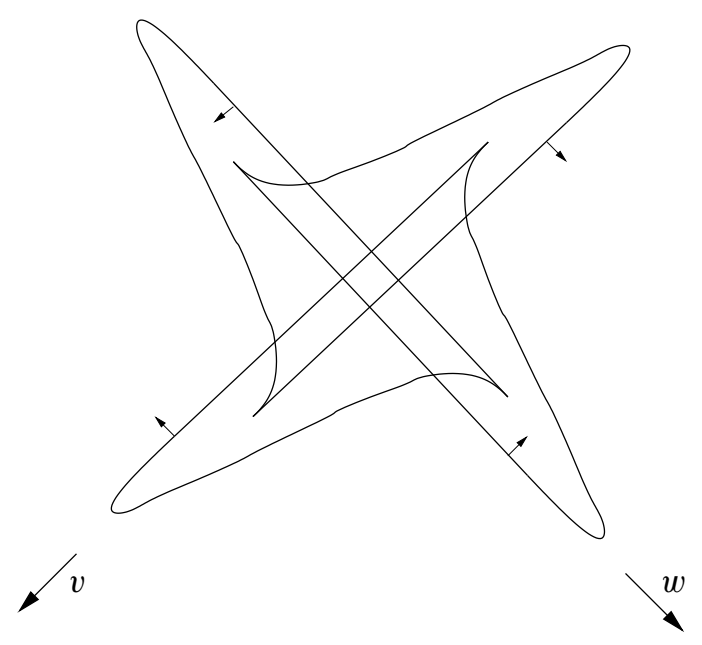

Figure 1: Middle stage of a sphere eversion

to connect these pieces with the band $B$ to form an immersed sphere we consider the local model of an immersion projecting with a cusp. If $(u, v)$ are local coordinates around a point in $S^{2}$ and $(x, y, z)$ are coordinates in $\mathbb{R}^{3}$ we have

$$
\begin{aligned}
& x(u, v)=u, \\
& y(u, v)=v\left(v^{2}+u\right), \\
& z(u, v)=v .
\end{aligned}
$$

The fold curve of the projection to the $x y$-plane is given by $\left\{(u, v): u=-3 v^{2}\right\}$. The projection of the curve $\left\{(u, v): u=-3 v^{2}+\epsilon\right\}$ to the $x y$-plane is a curve with a kink and one transverse double point for $\epsilon>0$, as $\epsilon \rightarrow 0$ the kink shrinks, for $\epsilon=0$ the curve has a cusp, and for $\epsilon<0$ the curve is injective. Using this model around the small kinks of the curves $\pi_{v} \circ h\left(\partial D_{n}\right)$ and $\pi_{v} \circ h\left(\partial D_{s}\right)$, see Figure 2, it is straightforward to connect $h\left(D_{n}\right)$ and $h\left(D_{s}\right)$ with an immersion $h$ of $B$ so that $\pi_{v} \circ h$ is as in Figure 1.

In order to describe the sphere eversion $g_{t}$ it thus remains to connect the middle stage $g_{\frac{1}{2}}$ described above with standard spheres of opposite coorientations. To this end, we use an abstract argument (although it is straightforward to draw a sequence of somewhat complicated pictures).

Consider any sphere immersion $f: S^{2} \rightarrow \mathbb{R}^{3}$ such that the height function $f_{v}(p)=$ $\langle v, f(p)\rangle$ has exactly two non-degenerate critical points for some direction $v$. We say that such a critical point is positive (negative) if the direction of the coorienting normal 

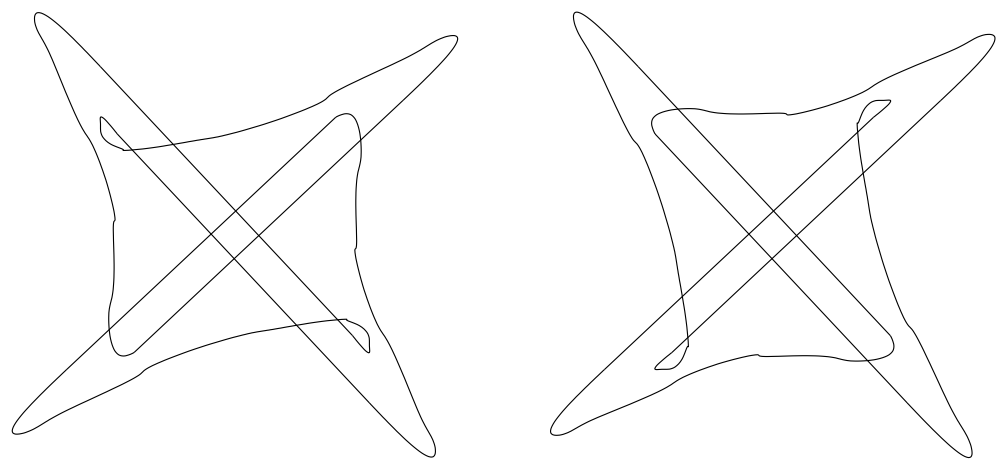

Figure 2: Two immersed disks

of the immersion agrees (does not agree) with its mean curvature vector at that point. Let the minimum of $f_{v}$ be $f_{v}\left(q_{-}\right)=m$ and the maximum be $f_{v}\left(q_{+}\right)=M$. Then for $m<a<M, f\left(f_{v}^{-1}(a)\right)$ is an immersed planar curve in a plane perpendicular to $v$. In this way we can view $f$ as a 1-parameter family of immersed planar curves which begins and ends at embedded circles. In particular, since the tangential degree of the members of such a family is constant, it follows that the two critical points in the direction $v$ have the same sign.

Lemma 2.3 Let $f_{j}: S^{2} \rightarrow \mathbb{R}^{3}$ be immersions and let $v_{j}$ be unit vectors, $j=0,1$. Assume that the height functions $\left(f_{j}\right)_{v_{j}}, j=0,1$, have exactly two non-degenerate critical points and that the signs of the critical points of $\left(f_{0}\right)_{v_{0}}$ agree with the signs of the critical points of $\left(f_{1}\right)_{v_{1}}$. Then there exists a regular homotopy $f_{t}: S^{2} \rightarrow \mathbb{R}^{3}$, $0 \leq t \leq 1$, from $f_{0}$ to $f_{1}$, and a continuous family $v_{t}, 0 \leq t \leq 1$, of unit vectors such that $\left(f_{t}\right)_{v_{t}}$ has exactly two non-degenerate critical points for all $t$.

Proof After composing $f_{0}$ with a rotation of $\mathbb{R}^{3}$ which takes $v_{0}$ to $v_{1}$, and a scaling and translation in the $v_{1}$-direction we may assume that $v_{0}=v_{1}=v$ and that the maxima (minima) of the functions $\left(f_{0}\right)_{v}$ and $\left(f_{1}\right)_{v}$ both equal $M(m)$. The lemma is then a consequence of the Smale-Hirsch $h$-principle for immersed planar circles as follows. We view the two immersions as two paths $F_{0}: S^{1} \times[0,1] \rightarrow \mathbb{R}^{2}$ and $F_{1}: S^{1} \times[0,1] \rightarrow \mathbb{R}^{2}$ of immersed plane curves, where the curve $F_{j}(\bullet, \tau)$ is $f_{j}\left(\left(f_{j}\right)_{v}^{-1}(m+\delta+\tau(M-\right.$ $m-2 \delta))), j=0,1$, for some small $\delta>0$. Then the start- and end-curves $F_{j}(\bullet, 0)$ and $F_{j}(\bullet, 1), j=0,1$, are very close to simple convex planar curves since they are intersections of the original immersions with planes perpendicular to the direction of the height function very close to its extrema. The Smale-Hirsch $h$-principle says that these paths of curves can be extended to a family of plane curve immersions 
$F: S^{1} \times[0,1]^{2} \rightarrow \mathbb{R}^{2}$ with $F(\bullet, \bullet, j)=F_{j}, j=0,1$, if and only if the corresponding paths $\vec{F}_{j}: S^{1} \times[0,1] \rightarrow \mathbb{R}^{2}-\{0\}$, which assign to a point $(\theta, t) \in S^{1} \times[0,1]$ the non-zero tangent vector $\frac{\partial}{\partial \theta} F_{j}(\theta, t)$, can be similarly extended as a continuous map $\vec{F}$ into $\mathbb{R}^{2}-\{0\}$.

Such a family $F: S^{1} \times[0,1]^{2} \rightarrow \mathbb{R}^{2}$ of plane curves can be used in an obvious manner to construct a regular homotopy $f_{t}: S^{2} \rightarrow \mathbb{R}^{3}, 0 \leq t \leq 1$, which connects $f_{0}$ to $f_{1}$ and which has properties as claimed. In order to finish the proof of the lemma we thus need only show that the topological extension problem has a solution. This is straightforward: consider $S^{1}$ as a union of a 0 -cell $e_{0}$ and a $1-$ cell $e_{1} \approx[0,1]$ with both endpoints identified with $e_{0}$. First define an extension of $\vec{F}$ on $e_{0} \times\{j\} \times[0,1]$ connecting $\vec{F}_{0}\left(e_{0}, j\right)$ to $\vec{F}_{1}\left(e_{0}, j\right), j=0,1$, in such a way that the loop $\vec{F}$ on $e_{0} \times \partial[0,1]^{2}$ is contractible. Then extend it to $\vec{F}: e_{0} \times[0,1]^{2} \rightarrow \mathbb{R}^{2}-\{0\}$. Since, $\vec{F}_{0} \mid e_{1} \times\{j\}$ is homotopic to $\vec{F}_{1} \mid e_{1} \times\{j\}, j=0,1$, we can define $\vec{F}$ also on $e_{1} \times\{j\} \times[0,1], j=0,1$. With this done $\vec{F}$ is defined on the boundary of $[0,1]^{3}$. Since $\pi_{2}\left(\mathbb{R}^{2}-\{0\}\right)=0$ we can find the desired $\vec{F}$ by extension over the cube.

With Lemma 2.3 established, we return to our sphere eversion $g_{t}$. If the immersion $g_{\frac{1}{2}}$ depicted in Figure 1 is sufficiently elongated in the $v$-direction then the resulting immersion has exactly two critical points in the $v$-direction which are both negative. Lemma 2.3 then implies that we can connect $g_{\frac{1}{2}}$ with the standard immersion with the outward coorientation. On the other hand, if $g_{\frac{1}{2}}$ is sufficiently elongated in the wdirection then the resulting immersion has exactly two critical points in the $w$-direction which are both positive and Lemma 2.3 implies that $g_{\frac{1}{2}}$ can be connected with the standard immersion with the inward coorientation as well. This gives a sphere eversion $g_{t}, 0 \leq t \leq 1$.

Proof of Theorem 1.1 We show that the sphere eversion $g_{t}$ described above can be carried out in such a way that $\kappa$ exceeds $8 \pi$ by an arbitrarily small amount. As shown in Figure 3, we can deform the middle stage depicted in Figure 1 keeping it an immersion until it is arbitrarily close to the degenerate middle stage depicted on the right in Figure 3. Furthermore, as the middle stage approaches the degenerate middle stage, it is clear from the picture that the total curvature of the fold curve approaches the sum of the angles marked on the degenerate middle stage (exterior angles at ordinary corners of the fold curve, interior angles at cusps). In Figure 4, we show how to connect the degenerate middle stage to an immersion which is very elongated in one direction in which it has only two local extrema. The angles contributing to the total curvature of the fold line of the degenerate immersion are easily seen to have sum equal to $4 \pi$ at the middle stage and to decrease toward $2 \pi$ as we rotate to obtain the elongated 

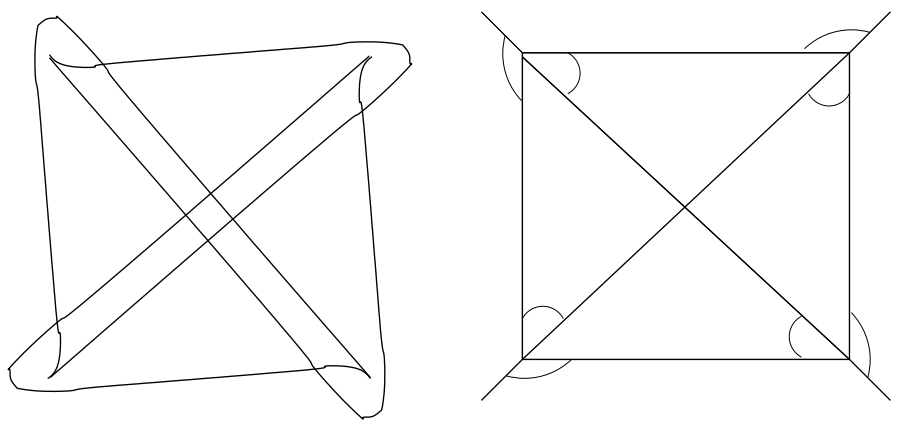

Figure 3: Deforming towards a degenerate middle stage

version on the left in Figure 4. It follows that we can connect two immersions $g_{0}$ and $g_{1}$ with exactly two extrema in directions $v$ and $w$, respectively, where $\langle v, w\rangle=0$, which are very elongated in their respective directions and such that the critical points of $g_{0}$ in direction $v$ have signs opposite to those of $g_{1}$ in direction $w$, by a regular homotopy $g_{t}, 0 \leq t \leq 1$, with $\max _{0 \leq t \leq 1} \kappa\left(g_{t}\right)<8 \pi+\epsilon$ for any $\epsilon>0$. To finish
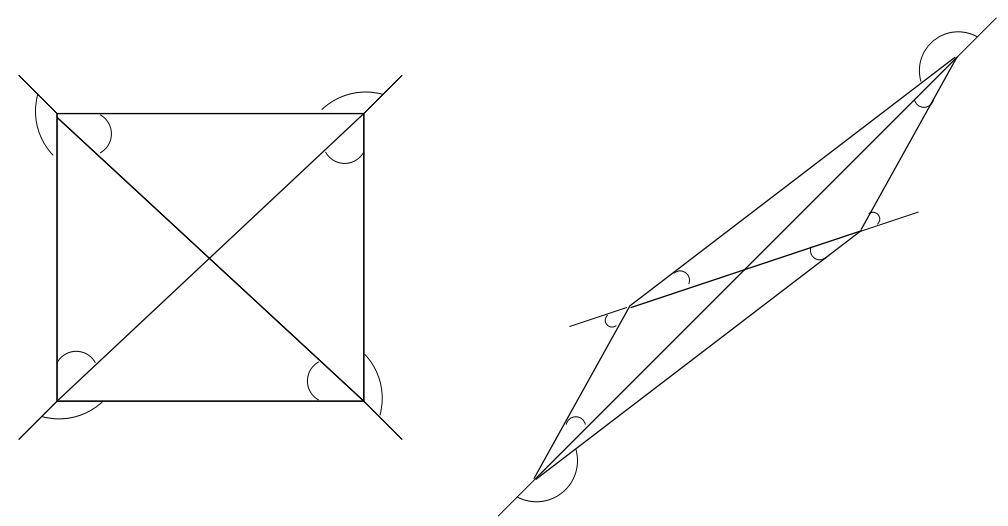

Figure 4: Deformation of the degenerate middle stage

the proof we note that Lemma 2.2 implies that $g_{0}$ and $g_{1}$ can be taken to have total curvature arbitrarily close to $4 \pi$. It will thus be sufficient to connect these immersions with standard spheres keeping $\kappa$ smaller than $8 \pi$. Regarding the elongated immersions with two extrema in the long direction as a family of immersed plane curves as in the proof of Lemma 2.3 it is not hard to show that it is possible to connect these to standard sphere keeping $\kappa$ close to $4 \pi$ by elongating the entire deformation in the direction 
where the spheres have only two extrema until we arrive at a sphere immersion which is the boundary of a convex body. The latter can then be connected to a standard sphere through boundaries of convex bodies.

\section{General properties of local minima}

In this section we discuss local minima of the total curvature functional in general dimensions using some tools from contact geometry.

\subsection{Basic contact notions}

A contact structure on an orientable $(2 n+1)$-manifold $N$ is a completely nonintegrable field of tangent hyperplanes $\xi \subset T N$. That is, a field of hyperplanes given as $\xi=\operatorname{ker} \alpha$, where the non-vanishing 1 -form $\alpha$ (the contact form) is such that the $(2 n+1)-$ form $\alpha \wedge(d \alpha)^{n}$ is a volume form on $N$. Note that if $\alpha$ is a contact form then $d \alpha \mid \xi$ is a symplectic form. A diffeomorphism of contact manifolds $(N, \xi) \rightarrow\left(N^{\prime}, \xi^{\prime}\right)$ is called a contactomorphism if it maps $\xi$ to $\xi^{\prime}$. An immersion of an $n$-manifold $f: L \rightarrow N$ is called Legendrian if $d f_{p}\left(T_{p} M\right) \subset \xi_{f(p)}$ for all $p \in L$.

\subsection{The Legendrian lift of an immersion}

Let $M$ be an $n$-manifold, let $T^{*} M$ be its cotangent bundle, and let $\pi: T^{*} M \rightarrow M$ be the projection. Define the $1-$ form $\theta_{M}$ on $T^{*} M$

$$
\theta_{M}(p) V=p(d \pi V), \quad p \in T^{*} M, V \in T_{p}\left(T^{*} M\right) .
$$

The unit cotangent bundle $U T^{*} \mathbb{R}^{n+1}$ of $\mathbb{R}^{n+1}$ carries a natural contact structure. Consider $U T^{*} \mathbb{R}^{n+1}$ as a subset of $T^{*} \mathbb{R}^{n+1}$, then the restriction $\alpha$ of the 1 -form $\theta_{\mathbb{R}^{n+1}}$ to $U T^{*} \mathbb{R}^{n+1}$ is a contact form. Also the 1 -jet space $J^{1}\left(\mathbb{S}^{n}, \mathbb{R}\right)=T^{*} \mathbb{S}^{n} \times \mathbb{R}$ of $\mathbb{S}^{n}$ carries a natural contact structure with contact 1 -form

$$
\beta=d z-\theta_{\mathbb{S} n}
$$

where $z$ is a linear coordinate on the $\mathbb{R}$-factor.

If $v \in \mathbb{R}^{n+1}$ then let $v^{*}$ be the linear form on $\mathbb{R}^{n+1}$ given by $\langle v, \cdot\rangle$, and if $p$ is a linear form let $p^{*}$ be the vector such that $p=\left\langle p^{*}, \cdot\right\rangle$. We write elements in $T^{*} \mathbb{R}^{n+1}$ as $(x, p)$, where $x \in \mathbb{R}^{n+1}$ and where $p$ is a linear functional on $\mathbb{R}^{n+1}$. Likewise, elements in $T_{v}^{*} \mathbb{S}^{n}$ will be written as $(v, p)$ where $v \in \mathbb{S}^{n} \subset \mathbb{R}^{n+1}$ and where $p$ 
is a linear functional on $\mathbb{R}^{n+1}$ such that $p(v)=0$. Note that the diffeomorphism $\Phi: U T^{*} \mathbb{R}^{n+1} \rightarrow J^{1}\left(\mathbb{S}^{n}\right)$

$$
\Phi(x, p)=\left(p^{*}, x^{*}-p(x) p, p(x)\right)
$$

is a contactomorphism.

If $f: M \rightarrow \mathbb{R}^{n+1}$ is an immersion of an oriented $n$-manifold we define its Legendrian lift $L f: M \rightarrow U T^{*} \mathbb{R}^{n+1}$ by

$$
L f(p)=\left(f(p), v(p)^{*}\right)
$$

where $v(p)$ is the positive normal of $f$. Using $\Phi$ we may regard $L f$ as a map into $J^{1}\left(\mathbb{S}^{n}\right)$ as well. Note that $L f$ is a Legendrian immersion.

Let $\pi_{1}: U T^{*} \mathbb{R}^{n+1} \rightarrow \mathbb{R}^{n+1}$ and $\pi_{2}: U T^{*} \mathbb{R}^{n+1} \rightarrow \mathbb{S}^{n}$ be the natural projections. If $\Gamma: M \rightarrow U T^{*} \mathbb{R}^{n+1}$ is a Legendrian immersion such that $\pi_{1} \circ \Gamma$ is an immersion then there exists an orientation on $M$ such that $\pi_{2} \circ \Gamma^{*}$ is the Gauss map of $\pi_{1} \circ \Gamma$.

Finally, let $\phi: \mathbb{S}^{n} \rightarrow \mathbb{S}^{n}$ be a diffeomorphism then $\phi$ has a natural lift to a contactomorphism $\Phi: J^{1}\left(\mathbb{S}^{n}\right) \rightarrow J^{1}\left(\mathbb{S}^{n}\right)$,

$$
\Phi(x, p, z)=\left(\phi(x), p \circ\left[d \phi^{-1}\right], z\right) .
$$

Note that $\Phi$ depends continuously on $\phi$.

\subsection{Shrinking volumes of maps and of Gauss maps}

Let $M$ be a closed $n$-manifold and let $f: M \rightarrow \mathbb{S}^{n}$ be any smooth map. By Sard's theorem the critical values of $f$ form a subset of $\mathbb{S}^{n}$ of measure zero. Assume that there exists a point $p \in M$ such that $\operatorname{rank}\left(d f_{p}\right)=n$. Then the volume $\operatorname{vol}(f)$ of the map $f$ satisfies $\operatorname{vol}(f)>0$. If $p \in \mathbb{S}^{n}$ is a regular value we say that the absolute multiplicity of $f$ at $p$ is the (finite by compactness) number of points in $f^{-1}(p)$. We prove two lemmas about decreasing volumes of maps.

Lemma 3.1 Assume that $f: M \rightarrow \mathbb{S}^{n}$ has two regular values (a non-value is a regular value of absolute multiplicity 0 ) of different absolute multiplicities. Then there exists a 1 -parameter family of diffeomorphisms $\phi_{t}: \mathbb{S}^{n} \rightarrow \mathbb{S}^{n}, 0 \leq t \leq 1$, with $\phi_{0}=\mathrm{id}$ and such that

$$
\operatorname{vol}\left(\phi_{t} \circ f\right)<\operatorname{vol}(f), \text { for } 0<t \leq 1 \text {. }
$$

Proof Consider first the 1-dimensional case. Let $p$ and $q$ be points in $\mathbb{S}^{1}$ of absolute multiplicity $m$ and $n$ respectively with $m<n$. Let $A \subset \mathbb{S}^{1}$ be the positively oriented 
arc connecting $p$ to $q$. Since the subspace of regular values in $\mathbb{S}^{1}$ is open and since the absolute multiplicity is locally constant on this subspace, there exists $\delta>0$ such that in a $\delta$-neighborhood of $\partial A$ in $A$ the multiplicity is constant. Let $B(x, r)$ denote the $r$-ball around $x$ and choose a smooth function $\phi: \mathbb{S}^{1} \rightarrow[0,1]$ with the following properties

- $\phi=0$ on a $\frac{1}{8} \delta$-neighborhood of $\mathbb{S}^{1}-A$,

- $\phi=1$ on $A-\left(B\left(p, \frac{1}{2} \delta\right) \cup B\left(q, \frac{1}{2} \delta\right)\right)$,

- $\frac{d \phi}{d s} \geq 0$ on $B(p, \delta)$ and $\frac{d \phi}{d s} \leq 0$ on $B(q, \delta)$.

For small $\theta>0$ consider the 1 -parameter family of diffeomorphisms $\phi_{t}: \mathbb{S}^{1} \rightarrow \mathbb{S}^{1}$

$$
\phi_{t}(x)=e^{i \theta \phi(x) t} x .
$$

If $\theta>0$ is sufficiently small then, for $t>0, \phi_{t}$ strictly decreases the length of the region where the multiplicity equals $n$ and strictly increases the length of the region where the multiplicity equals $m$. The lengths of all regions of other multiplicities are left unchanged. The lemma follows in the 1-dimensional case.

In the higher dimensional case. Let $p, q \in \mathbb{S}^{n}$ be regular values of multiplicities $m$ and $n$, respectively with $m<n$. Connect $p$ to $q$ by an oriented great circle arc $A \subset \mathbb{S}^{1} \subset \mathbb{S}^{n}$. Again, for sufficiently small $\delta>0$, the absolute multiplicity is constant on a $\delta$-neighborhood of $\partial A$. Consider a tubular neighborhood $T \approx \mathbb{S}^{1} \times D(\eta)$, with fiber disks $D(\eta)$ of radii $0<\eta<\delta$, of the great circle $\mathbb{S}^{1}$. Let $s$ be a coordinate on $\mathbb{S}^{1}$ and let $\phi(s)$ be a function as above. Let $g:[0, \eta] \rightarrow[0,1]$ be a non-increasing smooth function with $g(0)=1$ and $g=0$ on $\left[\frac{1}{2} \eta, \eta\right]$. Let $\psi: D(\eta) \rightarrow[0,1]$ be the function $\psi(\xi)=g(|\xi|)$. Let $(s, \xi) \in \mathbb{S}^{1} \times D(\eta)$ be coordinates on $T$. For small $\theta>0$ define the 1-parameter family of diffeomorphism $\phi_{t}: \mathbb{S}^{n} \rightarrow \mathbb{S}^{n}$ as $\phi_{t}=\mathrm{id}$ on $\mathbb{S}^{n}-T$ and

$$
\phi_{t}(s, \xi)=\left(e^{i \theta \phi(s) \psi(\xi) t} s, \xi\right),
$$

on $T$. Since the metric of $\mathbb{S}^{n}$ has the form

$$
d s^{2}+f(|\xi|) d \xi^{2}
$$

in $T$, it follows that, for $t>0, \phi_{t}$ strictly decreases the volume of the set of regular values of absolute multiplicity $n$, strictly increases the volume of the set of regular values of absolute multiplicity $m$, and leaves the volumes of all regions of other multiplicities invariant. The lemma follows. 
Lemma 3.2 Assume that $f: M \rightarrow \mathbb{S}^{n}$ is a smooth map and that there are local coordinates $x=\left(x^{\prime}, x^{\prime \prime}\right) \in \mathbb{R} \times \mathbb{R}^{n-1}$ around $p \in M$ and coordinates $y=\left(y^{\prime}, y^{\prime \prime}\right) \in$ $\mathbb{R} \times \mathbb{R}^{n-1}$ around $f(p) \in \mathbb{S}^{n}$ such that

$$
f\left(x^{\prime}, x^{\prime \prime}\right)=\left(\left(x^{\prime}\right)^{2 k}, x^{\prime \prime}\right), \text { for some } k>0 .
$$

Then there is a 1-parameter family of diffeomorphisms $\phi_{t}: \mathbb{S}^{n} \rightarrow \mathbb{S}^{n}, 0 \leq t \leq 1$, supported in a small neighborhood of $f(p)$, and a cut-off function $\psi(x)$ supported in a neighborhood of $x$ such that

$$
\operatorname{vol}\left(\phi_{t \psi} \circ f\right)<\operatorname{vol}(f), \quad \text { for } 0<t \leq 1 .
$$

Proof The lemma can be proved by an obvious modification of the proof of Lemma 3.1 .

Proposition 3.3 Let $f: M \rightarrow \mathbb{R}^{n+1}$ be an immersion which is a local minimum of $\kappa$ of normal degree $d$. Then $\kappa(f)=\operatorname{vol}\left(\mathbb{S}^{n}\right)(|d|+2 k)$, for some integer $k \geq 0$.

Proof Note that the Gauss map $v$ of $f$ is smooth and that any regular value has algebraic multiplicity $d$, and therefore absolute multiplicity $|d|+2 k$ for some $k \geq 0$. If the volume of the Gauss map is different from $\operatorname{vol}\left(\mathbb{S}^{n}\right)(|d|+2 m)$ for all integers $m$ then it follows that there are regular values of $v$ of different multiplicities. Let $\phi_{t}: \mathbb{S}^{n} \rightarrow \mathbb{S}^{n}$, $0 \leq t \leq 1$, be a family of diffeomorphisms as in Lemma 3.1 which shrinks $\operatorname{vol}(v)$. This family induces a family of contactomorphisms $\Phi_{t}: J^{1}\left(\mathbb{S}^{n}\right) \rightarrow J^{1}\left(\mathbb{S}^{n}\right)$, see (3-2), and hence of $U T^{*} \mathbb{R}^{n+1}$. Since the set of Legendrian immersions $\Gamma: M \rightarrow U T^{*} \mathbb{R}^{n+1}$ such that $\pi_{1} \circ \Gamma$ is an immersion is open, it follows that for $\epsilon>0$, sufficiently small, $\pi_{1} \circ \Phi_{t} \circ f, 0 \leq t \leq \epsilon$, is a regular homotopy of $f$ shrinking the total curvature. The proposition follows.

Proposition 3.4 If the Gauss map of $f: M \rightarrow \mathbb{R}^{n+1}$ has the form of the map in Lemma 3.2 then there exists a regular homotopy $f_{t}, 0 \leq t \leq 1$, with $f_{0}=f$ and such that

$$
\left.\frac{d}{d t} \kappa\left(f_{t}\right)\right|_{t=0}<0
$$

Proof Similar to the proof of Proposition 3.3.

\section{RIT immersions and curvature generic local minima}

In this section we discuss relatively isotopy tight (RIT) immersions. These are closely related to local minima of the total curvature functional. We prove a non-injectivity result for RIT immersions and apply it to demonstrate Theorem 1.2. 


\subsection{Relatively isotopy tight immersions}

Let $M$ be a closed surface and recall that if $f: M \rightarrow \mathbb{R}^{3}$ and $v \in \mathbb{S}^{2}$ then $f_{v}$ denotes the height function in direction $v$ composed with $f$.

Definition 4.1 An immersion $f: M \rightarrow \mathbb{R}^{3}$ is relatively isotopy tight $(R I T)$ if

$$
\kappa(f)=2 \pi \max _{v \in \mathbb{S}^{2}} \mu\left(f_{v}\right)=2 \pi \min _{v \in \mathbb{S}^{2}} \mu\left(f_{v}\right) .
$$

and

$$
\min _{v \in \mathbb{S}^{2}} \mu\left(g_{v}\right) \geq \frac{\kappa(f)}{2 \pi},
$$

for every immersion $g$ in some neighborhood of $f$.

This is Definition A, in Kuiper and Meeks [6], where the following structure theorem appears as Fundamental Lemma 2. (For simplicity of formulation we state it for embeddings, the obvious analog for immersions holds as well.)

Theorem (Kuiper and Meeks) If $f: M \rightarrow \mathbb{R}^{3}$ is an RIT $C^{2}$-embedding (immersion) then there exists an integer $k$ and convex surfaces $\partial B_{0}, \ldots, \partial B_{k}$ in $\mathbb{R}^{3}$ such that the set $M_{K>0}=K^{-1}(0, \infty)$ satisfies

$$
M_{K>0}=\bigcup_{j=0}^{k}\left(\partial B_{j}\right)_{K>0} .
$$

The unique principal component $M_{j}^{+}$of $\partial B_{j} \cap M$ contains $\left(\partial B_{j}\right)_{K>0}$ and is obtained from $\partial B_{j}$ by deleting disjoint plane convex disks in $\partial B_{j}$. The boundary of such a disk is called a top-circle. The plane $\Pi(\gamma)$ of a top-circle $\gamma$ supports some neighborhood $U \subset M$ of $\Pi(\gamma) \cap M_{j}^{+}$. Moreover,

$$
\kappa(f)=4 \pi(1+g(M)+2 k) .
$$

All these properties together are sufficient for an embedding (immersion) to be RIT.

\subsection{Non-injectivity}

In [6], the following question concerning RIT immersions of 2-spheres is posed as Problem 5: Is there an RIT embedding of the 2 -sphere with $\kappa=4 \pi+8 \pi k$ for some $k>0$ ?, and it is shown that there are no such embeddings for $k=1$. In this subsection we prove the following result which give some partial information about this question (and which, as we shall see in Section 4.3, also leads to a proof of Theorem 1.2). 
Theorem 4.2 Let $f: S^{2} \rightarrow \mathbb{R}^{3}$ be an RIT immersion with the following properties.

(a) At least one component of $K^{-1}((-\infty, 0])$ which intersects $K^{-1}((-\infty, 0))$ is an annulus with one boundary component bounding a disk $D \subset S^{2}$ such that $K \geq 0$ on $D$.

(b) If $v$ is a non-vanishing normal vector field of $f$ and if $p, q \in S^{2}$ are any two points with $K(p)>0$ and $K(q)>0$ then $\langle v(p), H(p)\rangle \cdot\langle v(q), H(q)\rangle>0$, where $H(p)$ is the mean curvature vector of $f$ at $p$. (In other words the signs of $\langle v(p), H(p)\rangle$ agree for all $p \in S^{2}$ with $K(p)>0$.)

Then $f$ is non-injective. It follows in particular that every RIT immersion such that all components of $K^{-1}((-\infty, 0])$ are annuli is non-injective.

Theorem 4.2 will be proved using two lemmas which we present next. It should be viewed in the light of the structure theorem for RIT immersions which has the following consequences. Let $f: S^{2} \rightarrow \mathbb{R}^{3}$ be an RIT immersion. Then the structure theorem in Section 4.1 gives a subdivision of $S^{2}$ into planar surfaces of two kinds. The first kind is the planar surfaces $\Omega_{j}^{+}$which map in a one to one fashion onto the convex bodies $\partial B_{j}$ with planar convex disks removed. We write $\Omega^{+}$for the union $\cup_{j} \Omega_{j}^{+}$ over all components. The second kind of planar surface is a component of $S^{2}-\Omega^{+}$. We denote such components $\Omega_{k}^{-}$and their union $\Omega^{-}=\cup_{k} \Omega_{k}^{-}$. Then each $\Omega_{k}^{-}$is a sphere with $r \geq 2$ disks removed. Our first lemma gives a non-embeddedness condition for such components which are spheres with two disks removed.

Let $f: S^{1} \times[0,1] \rightarrow \mathbb{R}^{3}$ be an immersion with the following properties.

(i) The map $f \mid S^{1} \times\{a\}$ is a planar curve bounding a convex region $D_{a}$ in a plane $\Pi_{a}, a=0,1$,

(ii) for some neighborhood $N_{a}$ of $f^{-1}\left(\Pi_{a}\right), f\left(N_{a}\right)$ is supported by $\Pi_{a}$, and adjoining to $f$ a small collar in the plane $\Pi_{a}$, outside $D_{a}$, we get a $C^{1}-$ immersion, $a=0,1$, and

(iii) $\kappa(f)=4 \pi$.

The Gauss-Bonnet theorem then implies that $K \leq 0$ everywhere in $S^{1} \times[0,1]$. It follows from this that $f\left(S^{1} \times[0,1]\right)$ is contained in the convex hull of $\partial D_{0}$ and $\partial D_{1}$ (if not, it is straightforward to find a point $p \in f\left(S^{1} \times[0,1]\right)$ where $K>0$ ).

Lemma 4.3 If $D_{0} \cap D_{1} \neq \varnothing$, and if $\partial D_{0}$ and $\partial D_{1}$ are unlinked then $f$ is noninjective. 
Proof We have $D_{0} \cap D_{1} \neq \varnothing$ and $K \leq 0$ in $S^{1} \times[0,1]$. If $\partial D_{0} \cap \partial D_{1} \neq \varnothing$ then $f$ is not injective. Thus assume that $\partial D_{0} \cap \partial D_{1}=\varnothing$. The linking condition then implies that the boundary of one of the disks does not intersect the other. Assume for definiteness that $D_{0} \cap \partial D_{1}=\varnothing$.

We claim that $f\left(S^{1} \times[0,1]\right) \cap \operatorname{int}\left(D_{0}\right) \neq \varnothing$, where $\operatorname{int}(X)$ denotes the interior of $X$. Suppose that this is not the case. Then we complete $f\left(S^{1} \times[0,1]\right)$ to an embedding $g: D^{2} \rightarrow \mathbb{R}^{3}$ of the 2-disk $D^{2}$ as follows. Add a small collar $C_{0}(\eta)=\partial D_{0} \times[0, \eta]$ along $f\left(S^{1} \times\{0\}\right)$ in the direction of the normal $\nu_{0}$ of the plane $\Pi_{0}$ which points into the half space of $\mathbb{R}^{3}$ not containing $f\left(N_{0}\right)$. Also add a copy of $D_{0}, D_{0}(\eta)$ shifted $\eta$ units along this normal, and finally add a large annular region $A_{1}$ in $\Pi_{1}$ bounded by $\partial D_{1}$ and the boundary of a large disk containing $D_{1}$. This is the image of the piecewise smooth embedding $g: D^{2} \rightarrow \mathbb{R}^{3}$. (This is an embedding since $f\left(S^{1} \times[0,1]\right)$ lies inside the convex hull of its boundary.)

Let $h_{1}$ denote the height function in the direction perpendicular to $\Pi_{1}$ normalized so that $h_{1}=0$ on $\Pi_{1}$. We can further complete the embedding $g$ of the disk to embeddings of a sphere in two different ways: add the lower hemisphere $\left(h_{1} \leq 0\right)$ of the sphere containing $\partial A_{1}-\partial D_{1}$ as a great circle, or add the upper hemisphere $\left(h_{1} \geq 0\right)$ of this sphere. We call the former embedded sphere $G_{l}$ and the latter $G_{u}$.

Then $G_{u}$ and $G_{l}$ bound balls in $\mathbb{R}^{3}$. Noting that the global maximum on $G_{l}$ of $h_{1}$ and the global minimum on $G_{u}$ of $h_{1}$ lies in $D_{0}(\eta) \cup C_{0}(\eta)$ we find that the outward coorientations along $D_{0}(\eta)$ of both $G_{u}$ and of $G_{l}$ must point along $v_{0}$. This local coorientation however determines the outward coorientations of $G_{u}$ and $G_{l}$ along $A_{1}$. In particular, the outward coorientations of $G_{u}$ and $G_{l}$ along $A_{1}$ must agree. This, however, contradicts the fact that the global minimum of $h_{1}$ on $G_{l}$ and the global maximum of $h_{1}$ on $G_{u}$ are attained on the added half-spheres since their outward coorientations induce opposite coorientations on $A_{1}$. We conclude that $f\left(S^{1} \times I\right) \cap \operatorname{int}\left(D_{0}\right) \neq \varnothing$.

After an arbitrarily small translation of $D_{0}$ along the normal line to $D_{0}$ we may assume that $f$ intersects $\operatorname{int}\left(D_{0}\right)$ transversely. If some component of this intersection bounds a disk in $S^{1} \times[0,1]$, then this disk has an extremum in a direction perpendicular to $D_{0}$ and thus has a non-degenerate extremum in some direction arbitrarily close to this one. Such an extremum contradicts $K \leq 0$. On the other hand, if no component of the intersection bounds a disk in $S^{1} \times[0,1]$ then there exists a component which together with $\partial D_{0}$ bounds a cylinder which must have at least one extremum in the direction perpendicular to $D_{0}$, which again contradicts $K \leq 0$. We conclude that $f$ cannot be an embedding. 
Let $f: S^{1} \times[0,1] \rightarrow \mathbb{R}^{3}$ be an immersion with the properties (i)-(iii) (stated above Lemma 4.3) which is also an embedding. Let $v_{0}$ and $v_{1}$ be the unit normal vectors of $\Pi_{0}$ and $\Pi_{1}$ which point into the half spaces which do not contain $f\left(N_{0}\right)$ and $f\left(N_{1}\right)$, respectively. Moreover, assume that $\partial D_{0}$ and $\partial D_{1}$ are unlinked.

Lemma 4.4 If $v$ is a normal vector field of $f$ then for any $p \in S^{1} \times\{0\}$ and any $q \in S^{1} \times\{1\}\left\langle v_{0}, v(p)\right\rangle>0$ if and only if $\left\langle v_{1}, v(q)\right\rangle>0$.

Proof For small $\eta>0$, let $C_{j}(\eta)=\partial D_{j} \times[0, \eta]$ be the collar on $\partial D_{j}$ in direction $v_{j}$ and let $D_{j}(\eta)$ be $D_{j}$ shifted $\eta$ units along $v_{j}$. It follows from Lemma 4.3 that for $\eta>0$ small enough

$$
D_{0}(\eta) \cup C_{0}(\eta) \cup f\left(S^{1} \times I\right) \cup C_{1}(\eta) \cup D_{1}(\eta)
$$

is an embedded sphere. Considering the height functions in directions perpendicular to $\Pi_{0}$ and $\Pi_{1}$ we find that the outward coorientation of this sphere is $v_{0}$ along $D_{0}(\eta)$ and $v_{1}$ along $D_{1}(\eta)$. The lemma follows from the continuity of the coorientation.

Proof of Theorem 4.2 We argue by contradiction: assume we can find an RIT embedding $f$ with properties as described. Note first that if any two top-circles in the decomposition of $f$ are linked then $f$ cannot be an embedding. Assume thus these circles are pairwise unlinked.

Consider the image $f(D)$ of the disk $D$ where the curvature is non-negative, $D \subset \Omega_{+}$. Let $B$ denote the convex hull of $f(D)$. Let $A \subset \Omega_{-}$denote the non-positively curved cylinder in the decomposition of $f$ such that $\partial B \cap \partial A \neq \varnothing$. Write $\partial A=\Sigma_{1} \sqcup \Sigma_{2}$ and let $\Sigma_{1}=\partial B \cap \partial A$. Lemma 4.3 implies that the convex planar disk $\Delta_{2}$ bounded by $\Sigma_{2}$ must either lie entirely outside $B$ or entirely inside $B$.

If $\Delta_{2}$ lies outside $B$ then $A \cap \operatorname{int}\left(\Delta_{1}\right) \neq \varnothing$, where $\Delta_{1}$ is the convex planar disk bounded by $\Sigma_{1}$. (To see this, note that points in $A$ near $\Sigma_{1}$ lies inside $B$.) The argument used in the proof of Lemma 4.3 then shows there exists a point where $K>0$ in $A$. But $K \leq 0$ in $A$, hence $\Delta_{2}$ lies inside $B$.

As in the proof of Lemma 4.4 let, for small $\eta>0, C_{j}(\eta)=\partial \Sigma_{j} \times[0, \eta], j=1,2$, be a collar in the direction of the normal of the plane of $\Delta_{j}$ which points away from $A$ and let $\Delta_{j}(\eta)$ be $\Delta_{j}$ shifted $\eta$ units along this normal. Note that $f(D) \cup A \cup C_{2}(\eta) \cup \Delta_{2}(\eta)$ is an embedded 2 -sphere which subdivides $\mathbb{R}^{3}$ into two connected components. One of these components is a subset of $\operatorname{int}(B)$, we call that component $X(\eta)$.

Let $\Pi_{1}$ and $\Pi_{2}$ be the planes which contain $\Delta_{1}$ and $\Delta_{2}$, respectively. Consider an embedding $k: D^{2} \rightarrow \mathbb{R}^{3}$ with the following properties: 
(i) $k\left(\partial D^{2}\right)=\Sigma_{2}$,

(ii) there exists some neighborhood $U$ of $k^{-1}\left(\Pi_{2}\right)$ and some neighborhood $V$ of $\Pi_{1} \cap A$ in $A$ such that $k(U) \cup V$ is a smooth surface supported by $\Pi_{2}$, and

(iii) $k\left(\operatorname{int}\left(D^{2}\right)\right) \cap\left(f(D) \cup A \cup C_{2}(\eta) \cup \Delta_{2}(\eta)\right)=\varnothing$.

Clearly, such embeddings $k$ satisfies $k\left(D^{2}\right) \subset \overline{X(\eta)}$.

Let $E=S^{2}-\left(D \cup f^{-1}(A)\right)$. Then $f: E \rightarrow \mathbb{R}^{3}$ is a disk embedding which satisfies (i) and (ii). Moreover, for almost all $\eta>0, f(E)$ intersects $\Delta_{2}(\eta)$ transversely. We construct an embedding $g: D^{2} \rightarrow \mathbb{R}^{3}$ fulfilling also (iii) as follows.

By transversality $f(E) \cap \operatorname{int}\left(\Delta_{2}(\eta)\right)$ is a finite collection of circles. Consider an innermost circle $\gamma$ in this intersection and its preimage $\Gamma$ in $E$. Note that $\Gamma$ divides $E$ into a disk $E_{d}$ and an annulus $E_{a}$. Define the map $g^{\prime}: D^{2} \rightarrow \mathbb{R}^{3}$ by cutting $f(E)$ along $\gamma$ and replacing $f\left(E_{d}\right)$ with the disk bounded by $\gamma$ in $\Delta_{2}(\eta)$ shifted slightly in the direction of $f\left(E_{a}\right)$. By induction we remove all intersection circles. Let $g: D^{2} \rightarrow \overline{X(\eta)}$ denote the embedding constructed in this way.

Let $h_{2}$ be the height function in the direction perpendicular to $\Pi_{2}$ such that $h_{2}\left(\Pi_{2}\right)=0$, and such that $h_{2} \circ g$ is positive near the boundary of $D^{2}$. Then $h_{2} \circ g$ has a global minimum on $D^{2}$. Assume first that the value of $h_{2} \circ g$ at its global minimum equals 0 .

Note that $\Delta_{1}(\eta) \cup C_{1}(\eta) \cup A \cup C_{2}(\eta) \cup \Delta_{2}(\eta)$ is an embedded sphere which bounds a ball. Let $\alpha(\eta)$ be an arc inside this ball connecting $\Delta_{1}(\eta)$ to $\Delta_{2}(\eta)$ and meeting the boundary transversely. Add to $\alpha(\eta)$ two half-rays perpendicular to $\Delta_{1}(\eta)$ and $\Delta_{2}(\eta)$, respectively, to get an embedded curve $\beta(\eta)$. Note that $g\left(\partial D^{2}\right)$ and $\beta(\eta)$ have linking number one. Therefore $g\left(D^{2}\right)$ must intersect $\beta(\eta)$. In fact it must intersect $\alpha(\eta)$ since the global minimum of $h_{2} \circ g$ equals 0 . This however contradicts $k\left(D^{2}\right) \subset \overline{X(\eta)}$ for all $\eta>0$ since $\alpha(\eta) \in \mathbb{R}^{3}-\operatorname{int}(X(\eta))$.

It follows that the value at the global minimum of $h_{2} \circ g$ is smaller than 0 . This implies in particular that the global minimum on $g\left(D^{2}\right)$ of a height function $h$ very close to $h_{2}$ is attained at some point of $q$ of $g(D)$ with $K(q)>0$. Since the part of the image of $g$ which is not in the image of $f$ can be taken arbitrarily close to $\left\{h_{2}=0\right\}$ this non-degenerate global minimum $q$ must be a point in the image of $f$. Consider the embedded sphere $Y=f(D) \cup A \cup g\left(D^{2}\right)$. Since $q$ is a global minimum in $g\left(D^{2}\right)$ of a height function $h$ arbitrarily close to $h_{2}$ there exists a path in the closure of the bounded component of $\mathbb{R}^{3}-Y$ which connects $q$ to a point $q^{\prime} \in f(D)$ such that $h\left(q^{\prime}\right)<h(q)$ and such that $K\left(q^{\prime}\right)>0$. It follows that the outward coorientation $v$ of $Y$ satisfies

$$
\langle v(q), H(q)\rangle=-\left\langle v\left(q^{\prime}\right), H\left(q^{\prime}\right)\right\rangle .
$$


However, it is clear from the above construction that the coorientation $v$ of $Y$ agrees (up to an over all sign) with the coorientation of $f$ for points in $Y$ which are in the image of $f$. Thus, (4-1) contradicts our coorientation assumption on $f$ and we conclude that $f$ cannot be an embedding.

The last statement of the theorem is a direct consequence of the above and Lemma 4.4

\subsection{Curvature generic local minima}

Let $f: S^{2} \rightarrow \mathbb{R}^{3}$ be an immersion. Let $K$ denote the curvature function of the metric induced by $f$. We say that $f$ is curvature generic if 0 is a regular value of $f$.

Lemma 4.5 Let $f: S^{2} \rightarrow \mathbb{R}^{3}$ be a curvature generic local minimum of $\kappa$. Then $f$ is an RIT immersion such that any component $\Omega_{j}^{-}$of $K^{-1}(-\infty, 0]$, see Section 4.2 for notation, is a two-holed sphere.

Proof Let $\gamma \subset S^{2}$ be a component of $K^{-1}(0)$. Then $\gamma$ is an embedded circle. Let $s$ be a parameter along $\gamma$. We show that

$$
\frac{d}{d s} v(s)=0,
$$

where $v$ is the Gauss map of $f$. Assume not, then there exists an open arc $\alpha$ in $\gamma$ where $\frac{d}{d s} v \neq 0$. Since $f$ is curvature generic there exists coordinates $(u, v) \in(-\delta, \delta)^{2}=W$ around some point $p \in \alpha$ with $K^{-1}(0)=\{v=0\}$. Since $\frac{d}{d s} v(p) \neq 0$, the second fundamental form of $f$ is non-trivial at $p$ and hence has a non-zero eigenvalue. But since $K(p)=0$ at least one of the eigenvalues of the second fundamental equal zero. It follows that if $W$ is chosen small enough then the eigenvalues of the second fundamental form of $f$ are distinct throughout $W$. Let $e_{1}, e_{2}$ be the eigenvector fields of the second fundamental form along $W$, with corresponding eigenvalues $\lambda_{1}, \lambda_{2}$. Then $K=\lambda_{1} \lambda_{2}=0$ along $\{v=0\}$. Since 0 is a regular value of $K$ we find

$$
\left.\frac{\partial \lambda_{1} \lambda_{2}}{\partial v}\right|_{(u, 0)}=\left.\frac{\partial \lambda_{1}}{\partial v}\right|_{(u, 0)} \lambda_{2}(u, 0)+\left.\lambda_{1}(u, 0) \frac{\partial \lambda_{2}}{\partial v}\right|_{(u, 0)} \neq 0 .
$$

This implies that at most one of $\lambda_{1}, \lambda_{2}$ vanishes along $\{v=0\}$. Assume it is $\lambda_{1}$. Since $\frac{\partial}{\partial u} v(u, 0) \neq 0$ we see that $e_{1}$ and $\partial_{u}$ are linearly independent. Moreover $\frac{\partial \lambda_{1}}{\partial v}(u, 0) \neq 0$. Using the flow of $e_{1}$ we construct a coordinate system $(u, x)$ in a neighborhood of $\{v=0\}$. We have

$$
v(u, x)=v(u, 0)+\frac{\partial}{\partial x} v(u, 0) x+\frac{1}{2} \frac{\partial^{2}}{\partial x^{2}} v(u, 0) x^{2}+\mathcal{O}\left(x^{3}\right)
$$


Since $\frac{\partial}{\partial x} v(u, x)=\lambda_{1} e_{1}$ and $\frac{\partial^{2}}{\partial x^{2}} v(u, 0)=\frac{\partial \lambda_{1}}{\partial x} e_{1}$,

$$
v(u, x)=v(u, 0)++\frac{1}{2} \frac{\partial \lambda}{\partial x} e_{1}(u, 0) x^{2}+\mathcal{O}\left(x^{3}\right) .
$$

Thus $v$ has a fold singularity along $\{v=0\}$. By Proposition 3.4, this contradicts $f$ being a local minimum and we conclude that $\nu(s)=v_{\gamma}$ is constant along $\gamma$. Since $\left\langle\dot{\gamma}, v_{\gamma}\right\rangle=0$ we find that $\gamma$ lies in a plane orthogonal to $v_{\gamma}$.

We subdivide the components of $K^{-1}(0)$ into levels as follows. Any component $\gamma$ subdivides the sphere into two disks. If one of these disks do not contain other components of $\Omega_{0}=K^{-1}(0)$ we say that $\gamma$ is a curve of level 0 . In general we make the following inductive definition. Let $\Omega_{j}$ be the subset of $K^{-1}(0)$ obtained by removing from it all components of level smaller than $j$. Define a component of $K^{-1}(0)$ to have level $j$ if one of the two disks into which it divides $S^{2}$ does not contain any component in $\Omega_{j}$.

Let $\gamma$ be a $0-$ level component of $K^{-1}(0)$. By the above $f(\gamma)$ is planar curve in a plane with normal $v_{\gamma}$. We claim that $f(\gamma)$ must be convex. Consider an arc $A$ of $\gamma$. The union of one piece of the surface near $A$ (the positively curved one) and a planar region with boundary $A$ must be convex. It is clear that this piece lies in the direction of the curvature vector of $A$. Hence, since $\gamma$ separates negative curvature from positive (because 0 is a regular value of $K$ ), it must be locally convex and the plane in which it lies is a local support plane of $f$. Moreover, restricted to any curve sufficiently near $\gamma$ the image of the Gauss map is a curve wrapping around $v_{\gamma} \in \mathbb{S}^{2} n$ times. Filling this curve with a disk with singularity in the middle we get a branched cover of the sphere by a sphere with one singular point of multiplicity $n$. The Riemann-Hurwitz formula gives

$$
\chi\left(S^{2}\right)=n \chi\left(S^{2}\right)-(n-1)=n+1,
$$

where $\chi$ denotes the Euler characteristic. Hence $n=1$ and $f(\gamma)$ is convex.

Assume inductively that the images of all curves of level $j-1$ are planar and convex. Consider a level $j$ curve $\partial R_{+}$which bounds a region $R \subset S^{2}$ and such that all other boundary components $\partial R_{-}$of $R$ are curves of level $j-1$. Consider the Gauss map restricted to $R$. Our inductive assumption shows that the map $v \mid\left(R-\partial R_{+}\right)$ gives an immersion from the disk $\bar{R}$ obtained from $R$ by filling each component of $\partial R_{-}$with a disk. Arguing as above we find that $f\left(\partial R_{+}\right)$is convex as well and that $\kappa(R)=\operatorname{area}(v(\bar{R}))=4 \pi$. Thus by the Gauss-Bonnet theorem, if $h$ denotes the number of holes in the negatively curved sphere with holes

$$
\int_{R} K d A=-4 \pi=2 \pi(2-h)-2 \pi h=4 \pi-4 \pi h .
$$


Hence $h=2$ and it follows that each negatively curved component is a cylinder. (One may see that each component of $K^{-1}((-\infty, 0))$ is a cylinder also by using $K<0$ and the line fields arising as asymptotic directions.)

Proof of Theorem 1.2 It follows from Lemma 4.5, that any curvature generic local minimum of $\kappa$ is an RIT immersion which satisfies the conditions of Theorem 4.2. The theorem then follows from Theorem 4.2.

\section{References}

[1] TE Cecil, P J Ryan, Tight and taut immersions of manifolds, Research Notes in Mathematics 107, Pitman (Advanced Publishing Program), Boston (1985) MR781126

[2] T Ekholm, Regular homotopy and total curvature I: circle immersions into surfaces, Algebr. Geom. Topol. 6 (2006) 459-492

[3] M Gromov, Partial differential relations, Ergebnisse series 9, Springer, Berlin (1986) MR864505

[4] M W Hirsch, Immersions of manifolds, Trans. Amer. Math. Soc. 93 (1959) 242-276 MR0119214

[5] NH Kuiper, Convex immersions of closed surfaces in $E^{3}$. Nonorientable closed surfaces in $E^{3}$ with minimal total absolute Gauss-curvature, Comment. Math. Helv. 35 (1961) 85-92 MR0124865

[6] NH Kuiper, W Meeks, III, Total curvature for knotted surfaces, Invent. Math. 77 (1984) 25-69 MR751130

[7] P Li, S T Yau, A new conformal invariant and its applications to the Willmore conjecture and the first eigenvalue of compact surfaces, Invent. Math. 69 (1982) 269-291 MR674407

[8] N Max, T Banchoff, Every sphere eversion has a quadruple point, from: "Contributions to analysis and geometry (Baltimore, Md., 1980)", Johns Hopkins Univ. Press, Baltimore, Md. (1981) 191-209 MR648465

[9] S Smale, A classification of immersions of the two-sphere, Trans. Amer. Math. Soc. 90 (1958) 281-290 MR0104227

[10] S Smale, The classification of immersions of spheres in Euclidean spaces, Ann. of Math. (2) 69 (1959) 327-344 MR0105117

Department of mathematics, USC

Los Angeles CA 90803, USA

tekholm@usc.edu

Received: 8 February 2005 Revised: 22 February 2006

Algebraic 83 Geometric Topology, Volume 6 (2006) 